\title{
TITLE:
}

\section{Wall boundary model for primitive chain network simulations}

\section{$\operatorname{AUTHOR}(\mathrm{S})$ :}

Okuda, Satoru; Inoue, Yasuhiro; Masubuchi, Yuichi; Uneyama, Takasi; Hojo, Masaki

\section{CITATION:}

Okuda, Satoru ... [et al]. Wall boundary model for primitive chain network simulations. JOURNAL OF CHEMICAL PHYSICS 2009, 130(21): 214907.

\section{ISSUE DATE:}

2009-01

URL:

http://hdl.handle.net/2433/109904

\section{RIGHT:}

Copyright 2009 American Institute of Physics. This article may be downloaded for personal use only. Any other use requires prior permission of the author and the American Institute of Physics. The following article appeared in JOURNAL OF CHEMICAL PHYSICS 130, 214907 (2009) and may be found at http://link.aip.org/link/JCPSA6/v130/i21/p214907/s1 


\title{
Wall boundary model for primitive chain network simulations
}

\author{
Satoru Okuda, ${ }^{1}$ Yasuhiro Inoue, ${ }^{1, a)}$ Yuichi Masubuchi, ${ }^{2}$ Takasi Uneyama, ${ }^{2}$ and \\ Masaki Hojo ${ }^{1}$ \\ ${ }^{1}$ Department of Mechanical Engineering and Science, Kyoto University, Kyoto 606-8501, Japan \\ ${ }^{2}$ Institute for Chemical Research, Kyoto University, Kyoto 611-0011, Japan
}

(Received 9 March 2009; accepted 1 May 2009; published online 4 June 2009)

\begin{abstract}
In condensed polymeric liquids confined in slit channels, the movement of chains is constrained by two factors: entanglement among the chains and the excluded volume between the chains and the wall. In this study, we propose a wall boundary (WB) model for the primitive chain network (PCN) model, which describes the dynamics of polymer chains in bulk based on coarse graining upon the characteristic molecular weight of the entanglement. The proposed WB model is based on the assumptions that (i) polymers are not stuck but simply reflected randomly by the wall, and (ii) subchains below the entanglement length scale behave like those in bulk even near the wall. Using the WB model, we simulate the dynamics of entangled polymer chains confined in slit channels. The results show that as the slit narrows, the chains are compressed in the direction normal to the wall, while they are expanded in the parallel direction. In addition, the relaxation time of the end-to-end vector increases, and the diffusivity of the center of mass decreases. The compression in the normal direction is a natural effect of confinement, while the expansion is introduced by a hooking process near the wall. The trends revealed that the relaxation time and diffusivity depend on the increase in friction due to an increased number of entanglements near the wall, which is also associated with the hooking process in the PCN model. These results are expected within the assumptions of the PCN model. Thus, the proposed WB model can successfully reproduce the effects of wall confinement on chains. (C) 2009 American Institute of Physics. [DOI: 10.1063/1.3140941]
\end{abstract}

\section{INTRODUCTION}

The dynamics of condensed polymeric liquids in confined geometries has been increasingly important for scientific and technological areas such as molding manufacturing, ${ }^{1}$ DNA sequence analysis, ${ }^{2}$ and synthetic biology. ${ }^{3}$ In confined geometries, it is well known that rheological properties of condensed polymeric liquids are dramatically changed due to interactions between polymers and walls. For example, conformation and mobility near walls are reported to be different from those in bulk. ${ }^{4}$

To clarify the mechanism of these peculiar behaviors, theoretical and experimental studies of the dynamics of chains in dilute polymeric liquids confined in slit channels have been performed in the past few decades, revealing that the confinement effect of the wall changes the equilibrium conformation of the chain and reduces its diffusivity. The scaling theory of Brochard and de Gennes ${ }^{5}$ and a selfconsistent mean field theory with the Kirkwood approximation by Harden and Doi $^{6}$ predicted a power law of the diffusivity with respect to the channel width. Their predictions are also consistent with recent multiscale modeling studies ${ }^{7}$ using a Brownian dynamics method with hydrodynamic interactions, which have improved our understanding of the dynamics of chain molecules confined to channels.

On the other hand, in the case of condensed polymeric liquids in a confined geometry, there has been no report about the dynamics of the polymer molecules. It is known

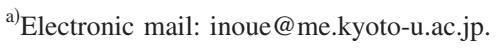

that the motions of polymer chains in condensed polymeric liquids are constrained by so-called entanglements. ${ }^{8}$ In the tube theory, ${ }^{8}$ entanglement is regarded as a geometrical constraint similar to a solid wall, although it has a completely different nature; constraint by an entanglement is dynamic, while that of the wall is static. Therefore, the coexistence of these two effects may introduce physical situations different from those of either effect alone. For example, the confinement effect of a wall on polymer chains may change the state of entanglements, which in turn can change the dynamics of the polymer chain. Thus, the dynamics of entangled polymer chains in confined geometries remains a challenging problem.

To investigate polymer dynamics and rheological properties numerically, particulate models such as coarse-grained molecular dynamics ${ }^{9}$ (MD) and dissipative particle dynamics $^{10}$ (DPD) have been employed. While DPD methods have made great contributions to analyzing the dynamics of unentangled polymers such as short polymers or dilute polymer solutions, they cannot handle entangled polymers because the chains slip past each other due to soft repulsive potentials. On the other hand, coarse-grained MD methods which employ the repulsive Lennard-Jones potential and the finite extensible nonlinear spring potential can successfully simulate entangled polymers. ${ }^{9}$ The coarse-grained MD was also used to study entangled polymers confined between walls and achieved success in analyzing and predicting physical properties of polymers such as the dependence of the gyration tensor on the distance between walls, the normal pressure, or the relaxation time. ${ }^{11}$ However, even by using 
the coarse-grained MD methods, simulations of wellentangled long polymer chains are numerically difficult because the longest relaxation time of such chains become quite long compared to the characteristic time scale of the coarse-grained MD. Thus to study well-entangled long polymers with reasonable computational costs, we need more coarse-grained mesoscopic models.

The primitive chain network (PCN) model, ${ }^{12}$ proposed some years ago, describes the kinetics of the topological constraint effect of entanglement by handling interactions of the entanglement between chains directly in three-dimensional (3D) space based on the idea of the slip link. The resulting dependence of mean-square radius of gyration, relaxation time, and diffusivity on the molecular weight has been shown to be consistent with the experimentally observed scaling laws. ${ }^{12}$ The PCN model has been applied for several polymers including the dynamics of linear polymers, ${ }^{12}$ branch polymers, ${ }^{13}$ and star polymers, to investigate the linear viscoelasticity. The PCN model has also been used for simulations of blend polymers and block copolymers, ${ }^{13,14}$ which have shown that it can reasonably reproduce the microphase formation process and the phase diagram for wellentangled copolymers. Thus, the PCN model is successful at simulating polymer melts. However, all these applications were limited to bulk because no wall boundary (WB) condition for the PCN model has been proposed yet. In this study, to simulate the dynamics of condensed polymeric liquids confined in slit channels, we develop a WB model for the PCN model.

In Sec. II, we briefly explain the PCN model. The WB model is proposed in Sec. III. In Sec IV, simulations using the WB model are described. Section V discusses the results. A summary of this paper is given in Sec. VI.

\section{PRIMITIVE CHAIN NETWORK MODEL}

Since minor differences exist among the several versions of PCN model already described in literature, here we briefly explain the model used in this study for clarity purposes. The PCN model represents each chain as a sequence of subchains, which connects consecutive entanglements. The entanglements are modeled as entanglement nodes consisting of two chains, each containing two subchains. The ends of the chains are modeled as end nodes containing a single subchain. Changes in the network topology are represented by hooking and unhooking, which occur only from the end of the chain.

Each entanglement node or chain-end node with the position vector, $\mathbf{R}$, obeys the Langevin equation,

$$
\zeta \dot{\mathbf{R}}=\sum_{j}, \frac{3 k T}{n_{j} b^{2}} \mathbf{r}_{j}-\nabla \mu+\mathbf{F},
$$

where $\Sigma_{j}^{\prime}$ means the sum over all subchains connected to the node directly. Here, $k T$ is the thermal energy, $b$ is the Kuhn monomer length, and $n_{j} \mathrm{~s}$ the number of monomers in the $j$ th subchain, respectively. $\mathbf{r}_{j}$ is a bond vector of the $j$ th subchain defined as a vector from an entanglement node or chain-end node to any one of the other connected nodes (see Fig. 1). (a)

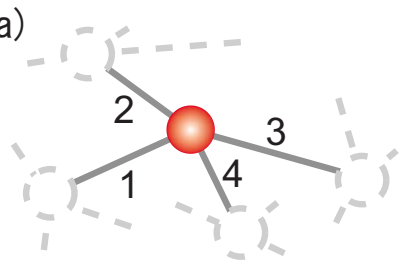

(b)

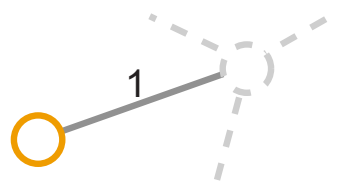

FIG. 1. (Color online) Schematic illustrations of subchains connected to (a) an entanglement node and (b) an end node. The entanglement node has four subchains, $j=\{1,2,3,4\}$, and the end node has a single subchain, $j=1$. Closed circle indicates the entanglement node, and open circle the end node. Solid segments indicate subchains connected to each node.

The left-hand side of Eq. (1) is the friction force on the node, where $\zeta$ indicates the friction coefficient of the node. In the model, we assume that the friction coefficient is proportional to the number of subchains connected to the node. $\zeta$ is defined as

$$
\zeta= \begin{cases}2 \zeta_{s} & \text { for entanglement } \\ \frac{1}{2} \zeta_{s} & \text { for chain end }\end{cases}
$$

Here, $\zeta_{s}$ is the friction coefficient of the subchain, which is taken to be of the same magnitude for all subchains. The first term on the right-hand side of Eq. (1) is the linear spring force due to entropic elasticity of subchains. The second term on the right-hand side of Eq. (1) is the field force introduced to account for the incompressible condition. Here, $\mu$ is the chemical potential as a function of the number of monomers derived from a free energy mentioned later. The third term, $\mathbf{F}$, on the right-hand side of Eq. (1) is a Gaussian random force obeying the following fluctuation-dissipation relations:

$$
\langle\mathbf{F}(t)\rangle=0, \quad\left\langle\mathbf{F}(t) \mathbf{F}\left(t^{\prime}\right)\right\rangle=2 \zeta k T \delta\left(t-t^{\prime}\right) \mathbf{1},
$$

where $\langle\ldots\rangle$ indicates the statistical average.

The rate of monomers sliding from one subchain to the next obeys the one-dimensional (1D) Langevin equation,

$$
\zeta_{s} \frac{a_{0}}{n_{0}} \dot{n}=\frac{3 k T}{b^{2}}\left(\frac{r_{i}}{n_{i}}-\frac{r_{i-1}}{n_{i-1}}\right)-\nabla \mu+f
$$

where $n_{0}$ and $a_{0}$ are the equilibrium number of monomer involved in subchain and equilibrium length of subchain respectively. The first term on the right-hand side of Eq. (4) is the elastic tension difference between the $i-1$ th and $i$ th subchains, along the chain. The third term, $f$, on the right-hand side of Eq. (4) is a Gaussian random force obeying the following fluctuation-dissipation relations in 1D,

$$
\langle f(t)\rangle=0, \quad\left\langle f(t) f\left(t^{\prime}\right)\right\rangle=2 \zeta_{s} k T \delta\left(t-t^{\prime}\right) .
$$

The chemical potential in Eqs. (1) and (4) is derived from a free energy density, $A$, as $\mu=\partial A / \partial n$. The fee energy density is defined as a function of the monomer density, $\phi$, as 


$$
\frac{A(\phi)}{k T}=\frac{1}{2} \varepsilon\langle\phi\rangle\left(1-\frac{\phi}{\langle\phi\rangle}\right)^{2},
$$

where $\varepsilon$ is the phenomenological modulus for incompressibility, and $\langle\phi\rangle$ is the average monomer concentration in the system. In the PCN model, the monomer density, $\phi$, is evaluated on a discretized regular mesh with a mesh size $a_{0}$. In other words, we calculate the monomer density in the $k$ th mesh, $\phi_{k}$, as the sum of all the monomers in the mesh such that

$$
\phi_{k}=\frac{1}{a_{0}^{3}} \sum_{l \in k} \sum_{j \in l}, \frac{n_{j}}{2},
$$

where the second summation in Eq. (7) is taken over the $j$ th subchain connected to the $l$ th node, and the first summation is taken over all the nodes in the $k$ th mesh. To calculate the thermodynamic forces, the monomer density, $\phi(\mathbf{r})$, at the position, $\mathbf{r}$, is approximated by $\phi_{k}$ of the $k$ th mesh to which $\mathbf{r}$ belongs.

The reconstruction of an entanglement network is represented by a rule for the addition and removal of entanglement nodes at chain ends. The rule is adopted every $\tau_{s}$ $=\zeta_{s} a_{0}^{2} / 3 k T$, which is the characteristic time of node diffusion, by monitoring the number of monomers in the end subchains. If this number falls below a certain lower threshold, $n / n_{0}<1 / 2$, the entanglement next to the examined subchain is removed. Conversely, if the number of monomers in the end subchains exceeds an upper threshold, $n / n_{0}>3 / 2$, an entanglement is newly created on the end subchain by hooking one of the surrounding subchains. The addition and removal events are dealt with in a way essentially similar to that of previous version of the PCN model. ${ }^{12}$

\section{WALL BOUNDARY MODEL}

In this section, we present a WB model for the PCN model. We focus on purely geometrical confinement effects of the wall on the polymer; namely, we suppose that the wall interacts with the polymers only in an excluded volume manner. In addition, we assume that (1) near-wall subchains are regarded as Gaussian, (2) near-wall nodes are thermally fluctuated the same as those in bulk, (3) the local structure of a subchain perturbed by reflection reaches equilibrium within the Rouse time of the subchain, and (4) thermal fluctuation dominates the movement of a reflected node within a certain time much less than the Rouse time of the subchain. These assumptions mean that polymer chains near the wall behave like those in bulk within the Rouse time of the subchain.

From assumptions 1-4, the node is scattered randomly from the point at which it collides. The probability density function, $f\left(\mathbf{x}, \Delta t_{d}\right)$, of a distance vector, $\mathbf{x}$, from the colliding point and a diffusing time, $\Delta t_{d}$, from the collision obeys the diffusion equation,

$$
\frac{\partial f\left(\mathbf{x}, \Delta t_{d}\right)}{\partial \Delta t_{d}}=D \frac{\partial f^{2}\left(\mathbf{x}, \Delta t_{d}\right)}{\partial \mathbf{x}^{2}},
$$

where $D$ is the diffusion coefficient of the node. The probability density, $f\left(\mathbf{x}, \Delta t_{d}\right)$, satisfies the following initial and boundary conditions:

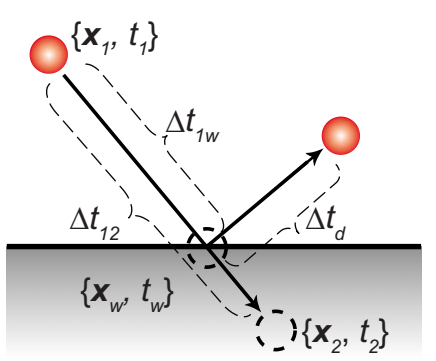

FIG. 2. (Color online) Schematic illustration of how to determine the diffusing time $\Delta t_{d}$ in the WB model. Arrows indicate the trajectory of the node (closed sphere). The node is reflected by the WB (solid line).

$$
\begin{aligned}
& f(\mathbf{x}, 0)=\delta(\mathbf{x}), \\
& \left.\frac{\partial f\left(\mathbf{x}, \Delta t_{d}\right)}{\partial \mathbf{x}} \cdot \mathbf{n}\right|_{\mathrm{WB}}=0,
\end{aligned}
$$

where we set the origin of the coordinates to the collision point. In Eq. (10), $\mathbf{n}$ is the unit vector normal to the wall. To solve Eq. (8), we decompose the probability density function into normal and parallel components,

$$
f\left(\mathbf{x}, \Delta t_{d}\right)=f_{\perp}\left(x_{\perp}, \Delta t_{d}\right) f_{\|}\left(x_{\|}, \Delta t_{d}\right) .
$$

Here, $x_{\perp}$ and $x_{\|}$represent the normal and parallel components of $\mathbf{x}$. Solving Eq. (8) with the initial and boundary conditions in Eqs. (9) and (10), the probability density functions are derived as

$$
\begin{aligned}
& f_{\perp}\left(x_{\perp}, \Delta t_{d}\right)=\frac{1}{\sqrt{\pi D \Delta t_{d}}} \exp \left(-\frac{\left(x_{\perp}-v_{\perp} \Delta t_{d}\right)^{2}}{4 D \Delta t_{d}}\right), \\
& f_{\|}\left(x_{\|}, \Delta t_{d}\right)=\frac{1}{\sqrt{4 \pi D \Delta t_{d}}} \exp \left(-\frac{\left(x_{\|}-v_{\|} \Delta t_{d}\right)^{2}}{4 D \Delta t_{d}}\right) .
\end{aligned}
$$

Here, $v_{\perp}$ and $v_{\|}$are the normal and parallel components, respectively, of the wall velocity vector.

Since the WB describes the behavior of the node at the wall, we should also define a mean field potential near the wall to solve the Langevin equations. In this study, we introduce the chemical potential of the node in the wall, $\mu^{\text {wall }}$, which includes the self-avoidance of the subchain due to the wall.

From assumptions 1-4, the diffusion coefficient should be equal to that in bulk within the Rouse time of the subchain. Thus, the diffusion coefficient can be expressed using the friction coefficient of the node,

$$
D=\frac{k T}{\zeta} .
$$

Here, we confirmed that the measured diffusion coefficient within the Rouse time of the subchain in periodic boundary simulations agreed with that estimated by Eq. (14). The excluded volume interaction between wall and polymer is modeled as the chemical potential of the wall, $\mu^{\text {wall }}$.

Figure 2 shows how to determine the diffusing time, $\Delta t_{d}$. We shall consider the time evolution of the nodes from time $t_{1}$ to time $t_{2}$ (we write $\Delta t_{12}=t_{1}-t_{2}$ ). The positions of the node 


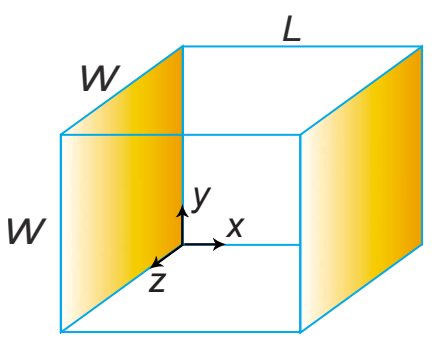

FIG. 3. (Color online) Schematic illustration of a simulation box confined in the slit channel. $x, y$, and $z$ are defined as displayed. A simulation box with a size of $L \times W \times W$ is confined by two parallel WBs, where $L$ is the slit width along the $x$ axis. Periodic boundaries are applied to the other boundaries.

at $t_{1}$ and $t_{2}$ are denoted by $\mathbf{x}_{1}$ and $\mathbf{x}_{2}$, respectively. We assume that the collision position, $\mathbf{x}_{w}$, is on a line between $\mathbf{x}_{1}$ and $\mathbf{x}_{2}$. Therefore, the elapsed time until the collision is estimated as $\left(\left|\mathbf{x}_{w}-\mathbf{x}_{1}\right| /\left|\mathbf{x}_{2}-\mathbf{x}_{1}\right|\right) \Delta t_{12}$. Thus, the rest time $\Delta t_{d}$ can be estimated as $\Delta t_{d}=\left\{1-\left(\left|\mathbf{x}_{w}-\mathbf{x}_{1}\right| /\left|\mathbf{x}_{2}-\mathbf{x}_{1}\right|\right)\right\} \Delta t_{12}$, and can be regarded as the diffusion time from the collision point. In Sec. IV, we simulate the dynamics of entangled chains confined in slit channels using the PCN model with the proposed WB model.

\section{SIMULATIONS}

Time unit is normalized by the relaxation time of subchains, $\tau_{s}$, distance by the equilibrium length of subchain, $a_{0}\left(=b n_{0}^{1 / 2}\right)$, number of monomers by the equilibrium number of monomers contained in a subchain, $n_{0}$, energy by $k T$, and friction by $\zeta_{s}$. The discretized forms of Eqs. (1) and (4) used for Euler integration become

$$
\begin{aligned}
& \tilde{\zeta} \Delta \widetilde{\mathbf{R}}=\Delta \widetilde{t}\left\{\sum_{j}, \frac{\widetilde{\mathbf{r}}_{j}}{\tilde{n}_{j}}-\frac{1}{3} \tilde{\nabla} \tilde{\mu}\right\}+\sqrt{2 \tilde{\zeta} \Delta \tilde{t} \mathbf{w},} \\
& \frac{\Delta \tilde{n}}{\tilde{\rho}}=\Delta \widetilde{t}\left\{\left(\frac{\widetilde{r}_{i}}{\tilde{n}_{i}}-\frac{\widetilde{r}_{i-1}}{\tilde{n}_{i-1}}\right)-\frac{1}{3} \tilde{\nabla} \tilde{\mu}\right\}+\sqrt{\frac{2}{3} \Delta \tilde{t} w},
\end{aligned}
$$

where tilde indicates the normalized value. $\mathbf{w}$ and $w$ are Gaussian random vectors in 3D and 1D, respectively, with zero mean and unit variance. The time step for integrating the Langevin equations, $\Delta \widetilde{t}$, is set as 0.01 . The reflection by the WB is performed after every update of positions in Eq. (15). The relative position vector of reflected node from the colliding position on the wall, $\widetilde{\mathbf{x}}$, is determined by Eqs. (12) and (13) as

$$
\begin{aligned}
& \tilde{x}_{\perp}=\sqrt{\frac{2 \Delta \tilde{t}_{d}}{3}} \widetilde{\zeta}|w|+\widetilde{v}_{\perp} \Delta \tilde{t}_{d}, \\
& \tilde{x}_{\|}=\sqrt{\frac{2 \Delta \tilde{t}_{d}}{3}} \widetilde{\zeta} w+\widetilde{v}_{\|} \Delta \tilde{t}_{d} .
\end{aligned}
$$

To reconstruct the entanglement network, the addition and removal of entanglement nodes are employed every unit time.

Simulation boxes with a size of $L \times W \times W$ are confined by parallel walls, where $L$ is the slit width and varies from $3 a_{0}$ to $32 a_{0}$ (see Fig. 3). Each chain has the number of mono- (a)
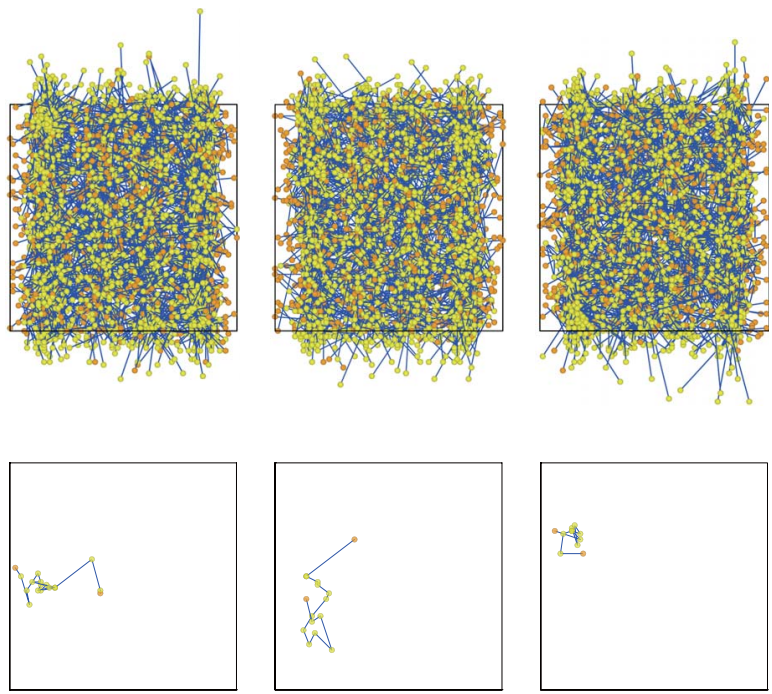

$\underset{z}{\stackrel{\gamma}{r} \rightarrow}$

100 step

200 step

300 step

FIG. 4. (Color online) Snapshots of (a) all and (b) single liner chains confined in a slit channel with $M=10 n_{0}$ and $L=8 a_{0}$. Red spheres indicate end nodes, yellow spheres entanglement nodes, and blue lines subchains.

mers, $M$, set to $5 n_{0}, 8 n_{0}, 10 n_{0}, 16 n_{0}$, and $20 n_{0}$. $W$ varies with respect to $M: W=6 a_{0}\left(M=5 n_{0}\right), W=8 a_{0}\left(M=8 n_{0}\right.$ and $\left.10 n_{0}\right)$, and $W=12 a_{0}\left(M=16 n_{0}\right.$ and $\left.20 n_{0}\right)$. The number density of monomers is set to $10 n_{0}$. The phenomenological modulus, $\varepsilon$, in Eq. (6) is set to 3. The chemical potential of the wall, $\mu^{\text {wall }}$, is set to $\mu(\langle\phi\rangle)$.

Figure 4 shows snapshots of a condensed polymeric liquid confined in a slit channel with $M=10 n_{0}$ and $L=8 a_{0}$. The figures show that the configuration of each chain in a condensed liquid is changed dynamically. To explain the effect of the wall on chain dynamics, we focus on three parameters: the projected components of radius of gyration, relaxation time of the end-to-end vector, and diffusivity of the center of mass of chains.

The position, $\mathbf{G}$, of the center of mass of each chain is defined as

$$
\mathbf{G}=\frac{1}{N_{t}} \sum_{i} \frac{n_{i}+n_{i+1}}{2} \mathbf{R}_{i},
$$

with the total number of monomers, $N_{t}$, in the chain. $\mathbf{R}_{i}$ and $n_{i}$ are the position vector and the number of monomers of the $i$ th node, respectively. The mean-square radius of gyration, $R_{g}^{2}$, is defined as

$$
R_{g}^{2}=\left\langle\frac{1}{N_{t}} \sum_{i} \frac{n_{i}+n_{i+1}}{2}\left(\mathbf{R}_{i}-\mathbf{G}\right)^{2}\right\rangle .
$$

Then, let $R_{i \alpha}$ be the component of the $\alpha$ axis of $\mathbf{R}_{i}$. The projected component normal to the walls, $R_{g \perp}$, and that parallel to the walls, $R_{g \|}$, are defined as

$$
R_{g \perp}^{2}=\left\langle\frac{1}{N_{t}} \sum_{i} \frac{n_{i}+n_{i+1}}{2}\left(R_{i x}-G_{x}\right)^{2}\right\rangle
$$

and 


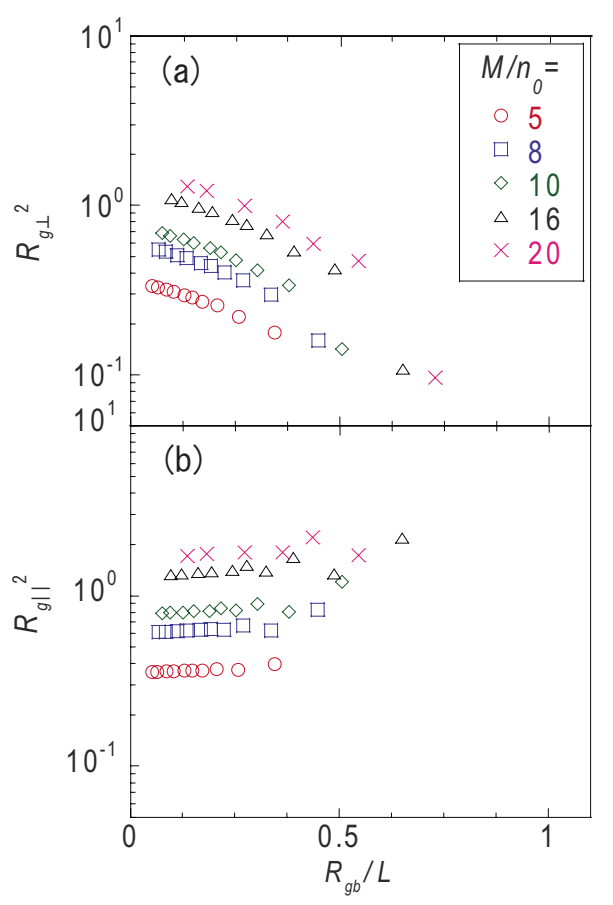

FIG. 5. (Color online) Variation of mean-square radius of gyration as a function of slit width, $L$. Mean-square radius of gyration is projected in the direction (a) normal, $R_{g \perp}$, and (b) parallel, $R_{g \|}$, to the wall. Gradually $R_{g \perp}$ decreases and $R_{g \|}$ increases.

$$
R_{g \|}^{2}=\left\langle\frac{1}{N_{t}} \sum_{i} \frac{n_{i}+n_{i+1}}{2} \frac{\left(R_{i y}-G_{y}\right)^{2}+\left(R_{i z}-G_{z}\right)^{2}}{2}\right\rangle,
$$

respectively.

Relaxation time is monitored through the autocorrelation function, $C_{\mathbf{P}}(t)$, of the end-to-end vector, $\mathbf{P}$, of each chain, given by

$$
C_{\mathbf{P}}\left(t-t_{0}\right)=\frac{\left\langle\mathbf{P}\left(t-t_{0}\right) \mathbf{P}\left(t_{0}\right)\right\rangle}{\left\langle\mathbf{P}\left(t_{0}\right) \mathbf{P}\left(t_{0}\right)\right\rangle} .
$$

In the long time region, $C_{\mathbf{P}}(t)$ is well described by a single exponential decay function, from which we obtain the longest relaxation time, $\tau_{\mathbf{P}}$.

In-plane diffusivity, $D_{\|}$, is calculated from the meansquare distance (MSD) of the center of mass of the chain,

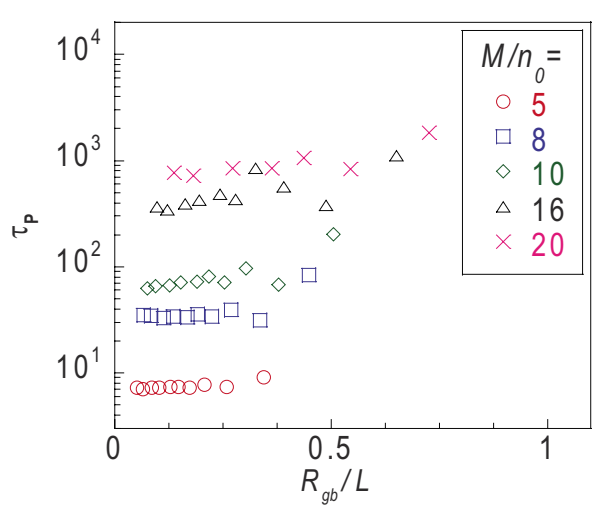

FIG. 6. (Color online) Plots of relaxation time $\tau_{\mathbf{P}}$ of the end-to-end vector as a function of $L . \tau_{\mathbf{P}}$ gradually increases as $L$ decreases.

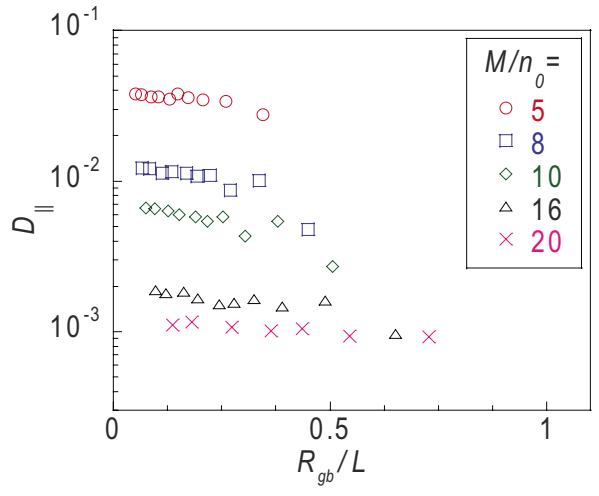

FIG. 7. (Color online) Plots of in-plain diffusivity $D_{\|}$of the center of mass as a function of $L . D_{\|}$gradually decreases as $L$ decreases.

$$
\begin{aligned}
\operatorname{MSD} & =\left\langle\left(G_{y}\left(t+t_{0}\right)-G_{y}\left(t_{0}\right)\right)^{2}+\left(G_{z}\left(t+t_{0}\right)-G_{z}\left(t_{0}\right)\right)^{2}\right\rangle \\
& =4 D_{\|} t .
\end{aligned}
$$

\section{RESULTS AND DISCUSSION}

Figures 5-8 show $R_{g \perp}, R_{g \|}, \tau_{\mathbf{P}}, D_{\|}$, and the number of entanglements, $Z$, respectively, as functions of the slit width, $L$, which is normalized by the average of the radius of gyration $R_{g b}$ calculated by the simulation with a box that is periodic in the $y$ and $z$ directions. The decrease in $R_{g \perp}$ shown in Fig. 5(a) can be understood as the confinement effect of the wall, which is reasonably attributed to the Neumann boundary condition. Although the trends of $R_{g \|}, \tau_{\mathbf{P}}$, and $D_{\|}$with respect to $L$ shown in Figs. 5(b) and 7 are similar to those of dilute liquid, the origin of these trends is based on different factors from dilute liquids. It is physically reasonable that these average parameters converge to the bulk values with increasing slit width.

The PCN model does not introduce excluded volume interactions, but it has the osmotic term to realize a certain uniformity of the network. Therefore, because the system box is applied with periodic boundaries except the walls, the osmotic pressure parallel to the walls must be statistically homogeneous in the system. Therefore, the force that expands chains parallel to the walls can be balanced with that of compressing the chains. Thus, the change in $R_{g \|}$ has no relation with that of $R_{g \perp}$, but is caused by another factor. The

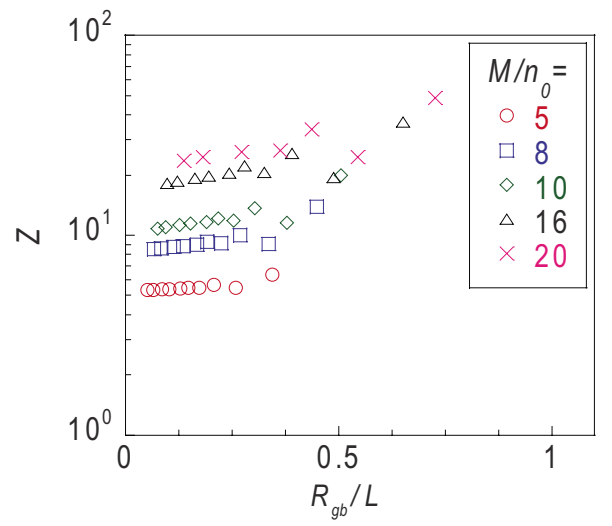

FIG. 8. (Color online) Plots of the mean number of entanglement $Z$ per single chain as a function of $L$. $Z$ gradually increases as $L$ decreases. 


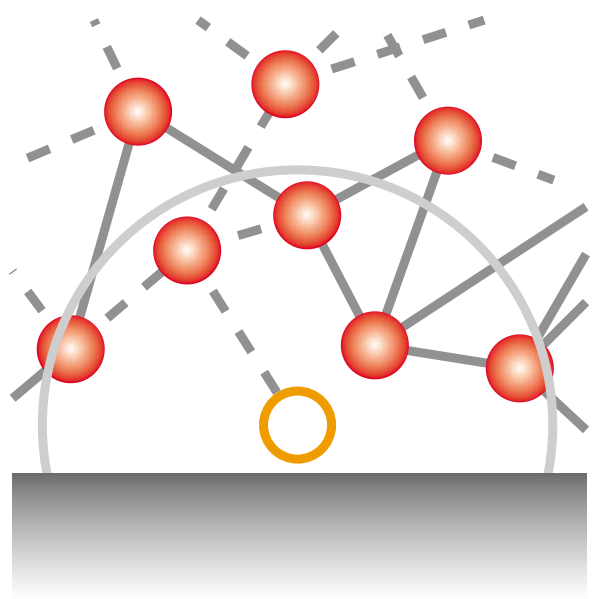

FIG. 9. (Color online) Schematic illustration of the process of creating entanglements near the wall. The hooking partner to create a new entanglement is sought in a sphere of radius $a$ (circle in the figure). The center of the figure is located at the end node of a chain (open yellow circle). Possible partners are all subchains in the sphere (solid segments). Other subchains are indicated as dashed segments. Apparently, the position of the newly created entanglement is closer to the bulk side than the wall side.

process of creating entanglements in the PCN model is a possible factor of the change in $R_{g \|}$. In the PCN model, an entanglement is newly created in a certain definite domain around the end of the chain. As schematically shown in Fig. 9, because the entropy of subchains decreases near the wall, the density of subchains near the wall becomes lower than that in the center of the channel, i.e., bulk hereafter. Thus, the position of newly created entanglements near the walls is in the bulk rather than near or on the wall. This elongates the average distance between the end of the chain and the new entanglement compared to that in bulk. The length of subchains connected to the new entanglement also becomes longer than that in bulk. Consequently, the radius of gyration of the chain near the wall increases. Although the walls do not confine the polymer chains in the parallel direction to the walls, the average radius of gyration in the parallel direction becomes larger by these elongated chains near the walls. Obviously, narrowing the slit enhances this apparent increase in $R_{g \|}$ because the contribution of the near-wall domain dominates that of the bulk in the narrower slit. Thus, the increase in $R_{g \|}$ is likely to be caused by the process of creating entanglements in the PCN model.

Figures 6 and 7 show that narrowing the slit width increases $\tau_{\mathbf{P}}$ and decreases $D_{\|}$. Although a decrease in $D_{\|}$is reported in the case of dilute polymeric liquid confined in a slit, the mechanism here seems to be different from that in the case of dilute polymers. According to scaling theory, polymer chains in dilute solutions can be modeled as selfavoiding chains of blobs, and the friction coefficient of a chain is proportional to the number of blobs. According to Ref. 1, narrowing the slit width increases the number of blobs, and thus decreases $D_{\|}$. In our condensed polymeric liquid model, the confinement effect on the mobility is mainly caused through entanglements because the friction of the entanglement determines the mobility of the chain. Figure 8 shows that narrowing the slit width increases the number of entanglements. In addition, because the PCN model
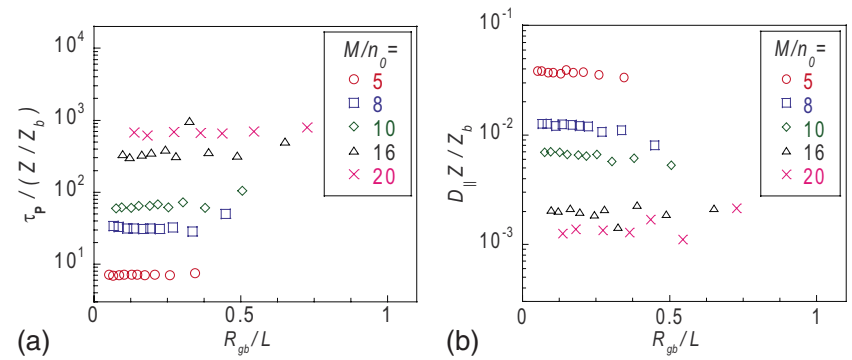

FIG. 10. (Color online) Scaling laws of the number of entanglement for the relaxation time and in-plain diffusivity. (a) Plots of the relaxation time scaled by $\tau_{\mathbf{P}} / Z$, and (b) plots of the in-plain diffusivity scaled by $D_{\|} Z$ as a function of $L$. $Z$ is normalized by the average number of entanglement, $Z_{b}$, calculated by the simulation with periodic boundaries in every direction.

used in this study assumes that the friction of the entanglements is constant independent of the density of monomers, the friction of the chain depends only on the number of entanglements. Thus, the increase in the number of entanglements directly relates to the decrease in $D_{\|}$and increase in $\tau_{\mathbf{P}}$ in this simulation.

Next, let us consider how the number of entanglements, $Z$, contributes to $D_{\|}$and $\tau_{\mathbf{P}}$. From the dimensional analysis, $D_{\|}$and $\tau_{\mathbf{P}}$ can be related to the friction of the chain,

$$
\tau_{\mathbf{P}} \propto \xi_{c},
$$

and

$$
D_{\|} \propto \xi_{c}^{1},
$$

respectively. Assuming that the friction coefficient is proportional to the number of entanglements, $\zeta_{c} \propto Z$, we obtain

$$
\begin{gathered}
\tau_{\mathbf{P}} \propto Z, \\
D_{\|} \propto Z^{-1} .
\end{gathered}
$$

Therefore, $\tau_{\mathbf{P}}$ and $D_{\|}$are scaled by $\tau_{\mathbf{P}} / Z$ and $D_{\|} Z$, respectively. Figure 10 supports these scaling laws.

It becomes apparent that the increase in the number of entanglements determines the mobility of chains. Next, let us consider why the number of entanglements is increased when the slit width narrows. Because reflections from the walls affect polymers near the wall only, we focus on the process of creating new entanglements near the wall (see Fig. 11). In the PCN model, a new entanglement is created near an end subchain when the number of monomers in the end subchain exceeds a threshold value. Thus, this addition rule involving monomer motions probably causes an increase in entanglements. Because there are few monomers near the wall, as mentioned earlier, the osmotic pressure gradient is directed to the wall. This osmotic pressure gradient slides monomers to the wall along the chain. However, because end subchains prefer to stay near the wall due to the recovery of entropy reduction, these end subchains create new entanglements using these monomers near the wall. Consequently, the number of entanglements increases near the wall. As the slit width narrows because the ratio of the near-wall domain to the entire system box becomes larger, the average number of entanglements increases. Thus, the observed increase in the number of entanglements is due to the nature of entangle- 


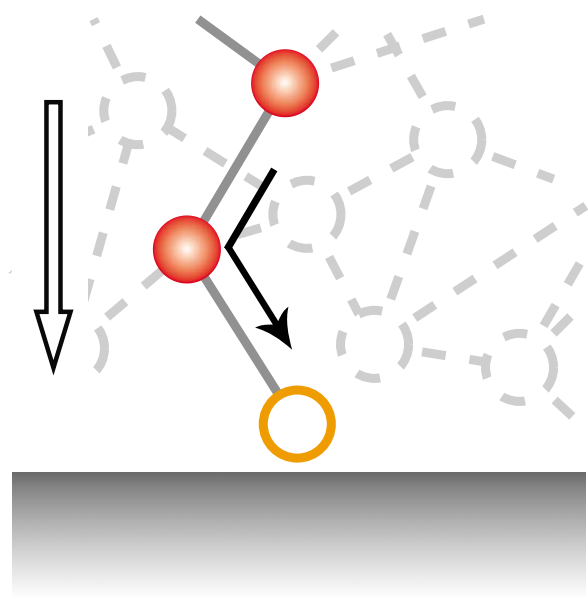

FIG. 11. (Color online) Schematic illustration of the increase in the number of monomers condensed in an end subchain, focusing on a chain with entanglement nodes (closed circles) and subchains (solid segments). Open circle indicates the end node of a chain located near the wall. All other nodes and subchains are indicated as dashed circles and lines, respectively. The osmotic pressure gradient is directed normal to the wall (open arrow). The number of monomers in the end subchain is increased by monomers in the near-wall chain sliding to the wall (closed arrow).

ment reconstruction in the PCN model, and hence it may not occur in reality. However, we shall avoid further modification of the PCN model at present because there have been no reports on addition of entanglement around the wall, to the authors' knowledge.

\section{CONCLUSION}

We developed the WB model for the PCN model to simulate the dynamics of condensed polymeric liquids confined in a slit channel. Simulations using the WB model show the following characteristics. When the slit width was decreased, $R_{g \perp}$ was reasonably decreased by the confinement effect of the wall on polymer chains. $R_{g \|}$ was increased by the expansion of chains due to the process of creating entanglements near the wall. As the slit width narrowed, $\tau_{\mathbf{P}}$ was increased and $D_{\|}$was decreased. These trends relative to the width could be explained by their dependence on the number of entanglements, $\tau_{\mathbf{P}} \propto Z$ and $D_{\|} \propto Z^{-1}$, because the friction of each chain was proportional to the number of entanglements. A possible factor affecting the increase in the number of entanglements was the process of creating entanglements in the near-wall domain.

To confirm whether the results are consistent with physical phenomena, we need to know the real trend of the number of entanglements. For example, we point to analyzing primitive passes of chains near the wall using more detailed simulations, such as the MD method. ${ }^{15}$ Even if the real trend might be different from our results, a possible modification is to tune the number of entanglements by altering the process of creating entanglement without changing the proposed WB model. ${ }^{16}$

\footnotetext{
${ }^{1}$ M. L. Buckleitner, Dubois and Pribble's Plastics Mold Engineering Handbook (Springer, New York, 1995).

${ }^{2}$ O. S. Andersen, Biophys. J. 77, 2899 (1999).

${ }^{3}$ J. H. Chen and N. C. Seeman, Nature (London) 350, 631 (1991).

${ }^{4}$ K. Tanaka, A. Taura, S. R. Ge, A. Takahara, and T. Kajiyama, Macromolecules 29, 3040 (1996); K. Tanaka, Y. Tsuchimura, K. Akabori, F. Ito, and T. Nagamura, Appl. Phys. Lett. 89, 061916 (2006); K. L. Ngai, A. K. Rizos, and D. J. Plazek, J. Non-Cryst. Solids 235-237, 435 (1998).

${ }^{5}$ F. Brochard and P. G. de Gennes, J. Chem. Phys. 67, 52 (1977).

${ }^{6}$ J. L. Harden and M. Doi, J. Phys. Chem. 96, 4046 (1992).

${ }^{7}$ R. M. Jendrejack, E. T. Dimalanta, D. C. Schwartz, M. D. Graham, and J. J. de Pablo, Phys. Rev. Lett. 91, 038102 (2003); R. M. Jendrejack, D. C. Schwartz, M. D. Graham, and J. J. de Pablo, J. Chem. Phys. 119, 1165 (2003); Y. L. Chen, M. D. Graham, J. J. de Pablo, G. C. Randall, M. Gupta, and P. S. Doyle, Phys. Rev. E 70, 060901 (2004); A. Balducci, P. Mao, J. Y. Han, and P. S. Doyle, Macromolecules 39, 6273 (2006).

${ }^{8} \mathrm{M}$. Doi and S. F. Edwards, The Theory of Polymer Dynamics (Clarendon, Oxford, 1986).

${ }^{9}$ K. Kremer and G. S. Grest, J. Chem. Phys. 92, 5057 (1990).

${ }^{10}$ R. D. Groot and P. B. Warren, J. Chem. Phys. 107, 4423 (1997).

${ }^{11}$ T. Aoyagi, J. Takimoto, and M. Doi, J. Chem. Phys. 115, 552 (2001).

${ }^{12}$ Y. Masubuchi, J. I. Takimoto, K. Koyama, G. Ianniruberto, G. Marrucci, and F. Greco, J. Chem. Phys. 115, 4387 (2001).

${ }^{13}$ Y. Masubuchi, G. Ianniruberto, F. Greco, and G. Marrucci, Modell. Simul. Mater. Sci. Eng. 12, S91 (2004).

${ }^{14}$ Y. Masubuchi, G. Ianniruberto, F. Greco, and G. Marrucci, J. Non-Cryst. Solids 352, 5001 (2006).

${ }^{15}$ S. K. Sukumaran, G. S. Grest, K. Kremer, and R. Everaers, J. Polym. Sci., Part B: Polym. Phys. 43, 917 (2005).

${ }^{16}$ T. Yaoita, T. Isaki, Y. Masubuchi, H. Watanabe, G. Ianniruberto, F. Greco, and G. Marrucci, J. Chem. Phys. 128, 154901 (2008).
} 